\title{
Title: Outcomes after successful fallopian tube recanalization- A large single institution experience: Observational retrospective study.
}

\author{
Joshua Marlow $^{1}$, Daniel Picus ${ }^{1}$, Jennifer Gould ${ }^{1}$, Sarah Connolly ${ }^{1}$, and Naganathan Mani ${ }^{1}$ \\ ${ }^{1}$ Washington University in Saint Louis School of Medicine
}

June 25, 2020

\begin{abstract}
Purpose: To determine outcomes after successful fallopian tube recanalization (FTR) in women who suffer infertility with documented tubal occlusion on hysterosalpingogram.(HSG) Methods: A retrospective review of consecutive successful FTR procedures from January 2010 to December 2016 was performed. 53 women who had HSG confirmed tubal occlusion from a single academic medical center and successful FTR were eligible for inclusion. 35 (66.0\%) patients had follow up at 12 months after FTR, with 13 conceiving within a year of the procedure. Complication, conception, and take-home-baby (THB) rates were recorded. The average age of patients was 32.3 years (range 26-42 years). All patients received peri- and postprocedure antibiotics. Results: Average follow-up after FTR was 1335 days. All patients tolerated the procedure well with no immediate complications. $13(37.1 \%)$ became pregnant after FTR. Of these women, 2 had ectopic pregnancies and 2 patients had spontaneous abortions. The THB rate after FTR for all patients was $25.7 \%$. The THB rate in women who became pregnant after FTR was $69.2 \%$. Of those women who did not become pregnant after FTR, 19 (84.6\%) went to assisted reproductive techniques, and of those, $8(42.1 \%)$ became pregnant. Conclusion: Infertility affects $8.4 \%$ of U.S. women, with tubal disease a major causative factor. In our study, successful FTR led to pregnancy in over a third of the patients with the majority giving birth to healthy babies. Given the success of obtaining pregnancy in combination with a low complication rate, FTR is a viable option in women who suffer from tubal infertility
\end{abstract}

\section{Introduction:}

Infertility, or in the inability to conceive after one year of unprotected intercourse under age 35 or six months of unprotected intercourse in women age 35 and older, affects approximately $6.0 \%$ of married U.S. women [1]. Infertility rates are higher in select patient populations including those with known risk factors for infertility including those who are of advanced age, endometriosis, malformation of the genitourinary system, and those who have a history of pelvic inflammatory disease. In $81 \%$ of women who suffer from infertility, there is an identifiable cause, with $11 \%$ having a tubal blockage [2].

Fallopian tubes are the conduit for the transportation of the oocyte into the uterus and when damaged prevent normal transport of the oocyte and sperm through the tube. The most common cause for tubal factory infertility is secondary to pelvic inflammatory disease associated with chlamydia or gonorrhea [3]. Diagnosis of tubal occlusion is often made via hysterosalpingogram (HSG). In one study, in women who were found to have proximal occlusion, $40 \%$ was caused by mucus plugging or debris, while another $20 \%$ was secondary to uterotubal spasm [3-5].

In women who have a distal occlusion, laparoscopy and reconstructive surgery is the treatment of choice, however, pregnancy rates were found to be $12 \%$ [6]. In women who are diagnosed with a proximal tubal occlusion, there are both surgical and non-surgical options. In women who have true cornual obstruction, 
tubocoronual anastomosis can be performed, however, often requires laparotomy, and intrauterine pregnancy rates range from 16-55\% and ectopic pregnancy rate from 7-30\% [7]. Alternatively, a nonsurgical option such as selective tubal catheterization, also known as fallopian tube recanalization (FTR), can be performed.

Current application of fallopian tube recanalization (FTR), was first described by Thurmond, et al in 1987. This procedure has the advantage of both being diagnostic via selective salpingography and in the event of tubal occlusion, therapeutic [8]. Historically, FTR has a technical success rate of $60-80 \%$ with intrauterine pregnancy rate of 20-60\%, with half achieving spontaneous pregnancy within one year [9-11]. Complication rates of FTR are low with studies demonstrating tubal perforation, infection, and ectopic pregnancy occurring from $1-9 \%$ [12-19].

Even with data demonstrating FTR is a safe and effective procedure for women who suffer from infertility secondary to proximal tubal occlusion, the procedure is underutilized [19]. Reasons for underutilization is multifactorial, but includes limited specific patient population, perceived technical difficulty, and radiation dose to the ovaries [19]. Given the limited data on this procedure, this study was performed to determine outcomes in a large cohort of patients in a tertiary medical center, after technically successful FTR in women who suffer infertility secondary to tubal occlusion.

\section{Methods:}

A retrospective study of consecutive technically successful FTR procedures from January 2010 through December 2016 was performed. The study was conducted at a single tertiary academic medical center. Women were included in this study if they met the following criteria: a hysterosalpingogram (HSG) confirmed tubal occlusion, a technically successful FTR, 1 year of follow-up after the procedure or became pregnant within the year after the FTR procedure. Technically successful FTR was defined as patency of the tube, as demonstrated by spontaneous spill of contrast into the peritoneal cavity, at the conclusion of the procedure after an intervention was performed.

Procedurally, similar methods were used as described previously by Rösch and Thurmond [20-21]. This included an HSG at the beginning of the procedure, identifying the obstruction, selective canalization of the fallopian tube with a catheter, use of hydrophilic glide wire to cross the obstruction, and concluding with a post-procedural HSG (Figure 1). All women received peri- and post-procedural antibiotics, with the most common regimen of doxycycline $100 \mathrm{mg}$ by mouth twice daily for 5 days, starting 2 days prior to the procedure.

In women who had technically successful FTR procedures and the inclusion criteria, the following data were recorded: complication, pregnancy, and take-home-baby (THB) rates. For purposes of this study, the THB rate was defined as the number of women who conceived and delivered a live baby. Additionally, sub-analysis of pregnancy rates was performed on women who did not conceive after a technically successful FTR and went onto assisted reproductive techniques.

This study was performed with local institutional review board approval.

\section{Results:}

53 women underwent technically successful FTR procedures between January 2010 and December 2016 (figure 2). Of these women, 18 did not have follow up at 1 year or did not conceive within 1 year after the procedure and, therefore, did not meet criteria for inclusion into this study. Of the remaining 35 women with technically successful FTR procedures, the average length of follow-up was 1335 days (224-2566 days). The average age of the woman at the time of the procedure was 32.3 (26-43 years).

1 peri-procedural complication occurred with an uncomplicated urinary tract infection 1 week after the procedure.

Post-procedurally, 13 women $(37.1 \%)$ had conceived naturally. Of these women, 2 women had ectopic pregnancies and 2 additional women had spontaneous abortions. The spontaneous intrauterine rate was 
$31.4 \%$. The THB rate for women who conceived naturally after a technically successful FTR procedure was $25.7 \%$.

In the 22 women who did not conceive after the FTR procedure, 19 women (86.4\%) elected to undergo assisted reproductive techniques (ARTs). Of which, 8 women (42.1\%) became pregnant.

\section{Discussion:}

In this study, women who underwent technically successful fallopian tube recanalization procedures, the spontaneous intrauterine pregnancy rate was $31.4 \%$, with the take-home-baby rate of $25.7 \%$. The spontaneous pregnancy rates in women with tubal disease without intervention is $7.9 \%$ [5]. Earlier studies on FTR demonstrated a spontaneous pregnancy rate of $58 \%$ [8]. These studies were limited, however, as strict patient selection was performed, which included both documented negative diagnostic laparoscopy and proximal tubal occlusion by HSG. In studies that lacked strict patient selection, such as lack of diagnostic laparoscopy, the spontaneous pregnancy rate decreased to $15-39 \%$, which is concordant to the results obtained in this study and what is likely to be seen in clinical practice $[8,21]$.

The ectopic pregnancy rate in this study was $5.7 \%$. Prior studies have documented ectopic pregnancy rates which have ranged from $0-13 \%[8,17,21]$. The slightly higher ectopic pregnancy rate found in this study could conceivably be secondary to lack of strict patient population selection (i.e. lack of diagnostic laparoscopy). A possible explanation for ectopic pregnancies after FTR has been thought to be secondary to peritubal adhesions, which is unable to be seen on FTR, however, could be readily identifiable on diagnostic laparoscopy [17]. Peri-procedural complication rate was low, with only one uncomplicated urinary tract infection identified, which resolved after a short course of oral antibiotics. An additional 2 women had spontaneous abortions. These rates do not appear significantly different when compared to literature [22].

There are some limitations to this study. As this study was conducted at an academic tertiary medical center in the Midwest of the United States, our study population may not be applicable universally. Additionally, the study size is relatively small with only 35 women meeting the inclusion criteria, however, this procedure is underutilized and not commonly performed.

Given the safety profile of fallopian tube recanalization and the improved pregnancy rate, fallopian tube recanalization is a simple, viable option which can be performed by interventional radiologists in women who suffer from infertility secondary to tubal occlusion.

\section{Teaching Points:}

- Fallopian tube recanalization is a safe and effective procedure for women who suffer from infertility secondary to proximal tubal occlusion, the procedure is underutilized

- In this study technically successful fallopian tube recanalization procedures, the spontaneous intrauterine pregnancy rate was $31.4 \%$, with the take-home-baby rate of $25.7 \%$.

- Fallopian tube recanalization is a simple, viable option which can be performed by interventional radiologists in women who suffer from infertility secondary to proximal tubal occlusion.

Disclosure of interests: None.Contribution to authorship: NM conceived this study, NM, DP planned the study. JM, NM wrote the draft, and NM, JM carried out the analysis. JG, DP, NM, and SC performed the procedures and assisted in revision of the manuscript.Details of ethics approval: Washington University Institutional Review Board - IRB ID \# 201808005 approved 8/23/2018Funding: NoneAcknowledgements: None

\section{References:}

1. Chandra A, Copen CE, Stephen EH. Infertility and impaired fecundity in the United States, 1982-2010: data from the National Survey of Family Growth. Natl Health Stat Report.2013Aug 14;(67):1-18.

2. WHO Scientific Group on Recent Advances in Medically Assisted Conception \& World Health Organization. (1992). Recent advances in medically assisted conception: report of a WHO scientific group 
[meeting held in Geneva from 2 to 6 April 1990]. World Health Organization.

3. Practice Committee of the American Society for Reproductive Medicine. Committee opinion: role of tubal surgery in the era of assisted reproductive technology. Fertil Steril. 2012 Mar;97(3):539-45. doi: 10.1016/j.fertnstert.2011.12.031. Epub 2012 Jan 29.

4. Sulak PJ, Letterie GS, Coddington CC, Hayslip CC, Woodward JE, Klein TA. Histology of proximal tubal occlusion. Fertil Steril. 1987 Sep;48(3):437-40.

5. Rasmussen F, Lindequist S, Larsen C, Justesen P. Therapeutic effect of hysterosalpingography: oil- versus water-soluble contrast media-a randomized prospective study. Radiology. 1991 Apr;179(1)75-8.

6. Patton PE, Williams TJ, Coulam CB. Results of microsurgical reconstruction in patients with combined proximal and distal tubal occlusion: double obstruction. Fertil Steril. 1987 Oct;48(4):670-4.

7. Helsa J. Laparoscopic management of distal tubal disease. Infertil Reprod Med Clinics North Am. $1997 ; 8: 425$.

8. Thurmond AS, Novy MJ, Uchida BT, Rosch J. Fallopian tube obstruction: selective salpingography and recanalization. Radiology 1987; 163:511-514.

9. Sankpal RS, Confino E, Matzel A, Cohen LS. Investigation of the uterine cavity and fallopian tubes using three-dimensional saline sonohysterosalpingography. Int J Gynaecol Obstet 2001; 73:125.

10. Mishell D, Stenchever M, Droegemueller W, Herbst A. Infertility, etiology, diagnostic evaluation, management and diagnosis. In: Comprehensive Gynecology, 3rd ed, Mosby-Year Book, Inc., St. Louis 1997. p.1113.

11. Papaioannou S, Afnan M, Girling AJ, et al. The effect on pregnancy rates of tubal perfusion pressure reductions achieved by guide-wire tubal catheterization. Hum Reprod 2002; 17:2174.

12. Thurmond AS, Novy M, Uchida BT, Rösch J. Fallopian tube obstruction: selective salpingography and recanalization. Work in progress. Radiology 1987; 163:511.

13. Thurmond AS. Selective salpingography and fallopian tube recanalization. AJR Am J Roentgenol 1991; 156:33.

14. Thurmond AS, Machan LS, Maubon AJ, et al. A review of selective salpingography and fallopian tube catheterization. Radiographics 2000; 20:1759.

15. Confino E, Friberg J, Gleicher N. Preliminary experience with transcervical balloon tuboplasty. Am J Obstet Gynecol 1988; 159:370.

16. Maubon A, Rouanet JP, Cover S, et al. Fallopian tube recanalization by selective salpingography: an alternative to more invasive techniques? Hum Reprod 1992; 7:1425.

17. Thurmond AS, Rösch J. Nonsurgical fallopian tube recanalization for treatment of infertility. Radiology 1990; 174:371.

18. Thurmond AS. Pregnancies after selective salpingography and tubal recanalization. Radiology 1994; 190:11.

19. Honoré GM, Holden AE, Schenken RS. Pathophysiology and management of proximal tubal blockage. Fertil Steril 1999; 71:785.

20. Rösch J, Thurmond AS, Uchida BT, Sovak M. Selective transcervical fallopian tube catheterization: technique update. Radiology. 1988 July;168(1):1-5.

21. Thurmond AS. Fallopian tube catheterization. Semin Intervent Radiol. 2008 Dec;25(4):425-31. doi: $10.1055 / \mathrm{s}-0028-1102995$. 
22. Hovsepian DM, Bonn J, Eschelman DJ, Shapiro MJ, Sullivan KL, Gardiner GA Jr. Fallopian tube recanalization in an unrestricted patient population. Radiology. 1994 Jan;190(1):137-40.

\section{Hosted file}

FTR figures only.docx available at https://authorea.com/users/336655/articles/462326-titleoutcomes-after-successful-fallopian-tube-recanalization-a-large-single-institutionexperience-observational-retrospective-study 